\title{
Modelling approach to simulate reductions in LDL cholesterol levels after combined intake of statins and phytosterols/-stanols in humans
}

\author{
Simone RBM Eussen ${ }^{1,2}$, Cathy JM Rompelberg ${ }^{2 *}$, Olaf H Klungel ${ }^{1}$ and Jan $\mathrm{CH}$ van Eijkeren ${ }^{2}$
}

\begin{abstract}
Background: To examine the effects on LDL cholesterol of the combined use of statins and phytosterols/-stanols, in vivo studies and clinical trials are necessary. However, for a better interpretation of the experimental data as well as to possibly predict cholesterol levels given a certain dosing regimen of statins and phytosterols/-stanols a more theoretically based approach is helpful. This study aims to construct a mathematical model to simulate reductions in low-density lipoprotein (LDL) cholesterol in persons who combine the use of statins with a high intake of phytosterols/-stanols, e.g. by the use of functional foods.

Methods and Results: The proposed model includes the cholesterol pool size in the liver and serum levels of very low-density lipoprotein (VLDL) cholesterol. Both an additional and a multiplicative effect of phytosterol/-stanol intake on LDL cholesterol reduction were predicted from the model. The additional effect relates to the decrease of dietary cholesterol uptake reduction, the multiplicative effect relates to the decrease in enterohepatic recycling efficiency, causing increased cholesterol elimination through bile. From the model, it was demonstrated that a daily intake of $2 \mathrm{~g}$ phytosterols/-stanols reduces LDL cholesterol level by about $8 \%$ to $9 \%$ on top of the reduction resulting from statin use. The additional decrease in LDL cholesterol caused by phytosterol/-stanol use at the recommended level of $2 \mathrm{~g} / \mathrm{d}$ appeared to be similar or even greater than the decrease achieved by doubling the statin dose.

Conclusion: We proposed a simplified mathematical model to simulate the reduction in LDL cholesterol after separate and combined intake of statins and functional foods acting on intestinal (re)absorption of cholesterol or bile acids in humans. In future work, this model can be extended to include more complex (regulatory) mechanisms.
\end{abstract}

Keywords: model, statins, phytosterols, phytostanols, LDL cholesterol, lipids, coronary heart disease

\section{Background}

Increased total cholesterol and low-density lipoprotein (LDL) cholesterol levels represent a major risk for atherosclerosis and coronary heart disease (CHD). Lipid-lowering drugs, of which the hydroxymethylglutarylcoenzyme A (HMG-CoA) reductase inhibitors (statins) have shown to be the most effective, reduce morbidity and mortality in patients with CHD [1-3]. Since the last decade of the $20^{\text {th }}$ century, more interest has been given

\footnotetext{
* Correspondence: Cathy.Rompelberg@rivm.nl

${ }^{2}$ National Institute for Public Health and the Environment, P.O. Box 1, 3720 BA Bilthoven, The Netherlands Full list of author information is available at the end of the article
}

to changing dietary habits, for example with the appearance of the so-called functional foods. Dairy products enriched with phytosterols/-stanols are one of the best known and most used functional foods to lower elevated total and LDL cholesterol levels. Phytosterols/-stanols are thought to compete with cholesterol for solubilisation into mixed micelles, the transport vehicles for cholesterol across the intestinal wall. Consequently, the intestinal (re)absorption of cholesterol is reduced, faecal output is increased and total and LDL cholesterol levels are lowered by $6 \%$ and $10 \%$, respectively $[4,5]$. Due to the rising public awareness of health and nutritional improvement, and the mounting evidence of the
Ciomed Central 
effectiveness of phytosterols/-stanols, it is conceivable that in the near future an increasing number of people will combine their statin therapy with these functional foods.

To examine the effects on total and LDL cholesterol levels of the combined intake of statins and phytosterols/-stanols, in vivo studies and clinical trials are necessary. However, for a better interpretation of the experimental data as well as to possibly predict cholesterol levels given a certain dosing regimen of statins and phytosterols/-stanols a more theoretically based approach is helpful.

The present study focuses on the combined effect of atorvastatin and phytosterols/-stanols. However, our model can easily be applied to other statins and similar acting functional foods (e.g. soluble dietary fibres) as well. Moreover, based on certain genetic variants associated with cholesterol absorption and production an individual's specific reduction in total and LDL cholesterol can be predicted.

\section{Methods}

We propose a simplified mathematical model to estimate reductions in LDL cholesterol after separate and combined intake of statins and phytosterols/-stanols (Figure 1). A list of model variables and abbreviations is presented in Table 1 . Since LDL is the product of very low-density lipoprotein (VLDL) delipidation and VLDL also transports, although to a lesser extent than endogenous triglycerides,[6] cholesterol from the liver to the blood circulation, our model includes the modelling of the metabolism of VLDL cholesterol as well. Also a hepatic cholesterol pool is accounted for in the model, because VLDL cholesterol secretion depends on cholesterol pool size. In the next section, we first describe a basic model which includes the modelling of the cholesterol pool, VLDL cholesterol and LDL cholesterol. Subsequently, this basic model is reformulated to express reductions in LDL cholesterol level dependent of statin and/or phytosterol/-stanol intake. Published scientific literature was used to estimate specific model parameters. In the second part of the present study (Results section), we tested the appropriateness of our model using available published experimental data.

\section{Basic cholesterol model}

\section{Modelling of the cholesterol pool}

A mass balance is considered with cholesterol input from endogenously produced cholesterol $P_{C}$ and from cholesterol taken up from the diet, $I_{C}$. Only a fraction $f_{a b s}$ of dietary cholesterol is assumed to be taken up across the gut wall, and consequently the uptake of dietary cholesterol is $U_{C}=f_{a b s} \cdot I_{C}$. The mass balance output consists of produced VLDL cholesterol, cholesterol cleared by elimination of excess cholesterol through bile and cholesterol needed to produce bile salts. For simplicity, we neglect the reverse cholesterol transport mediated by high-density lipoprotein (HDL) and the existence of a hepatic cholesteryl ester pool that might be involved in cholesterol homeostasis.

\section{Extrahepatic tissues}

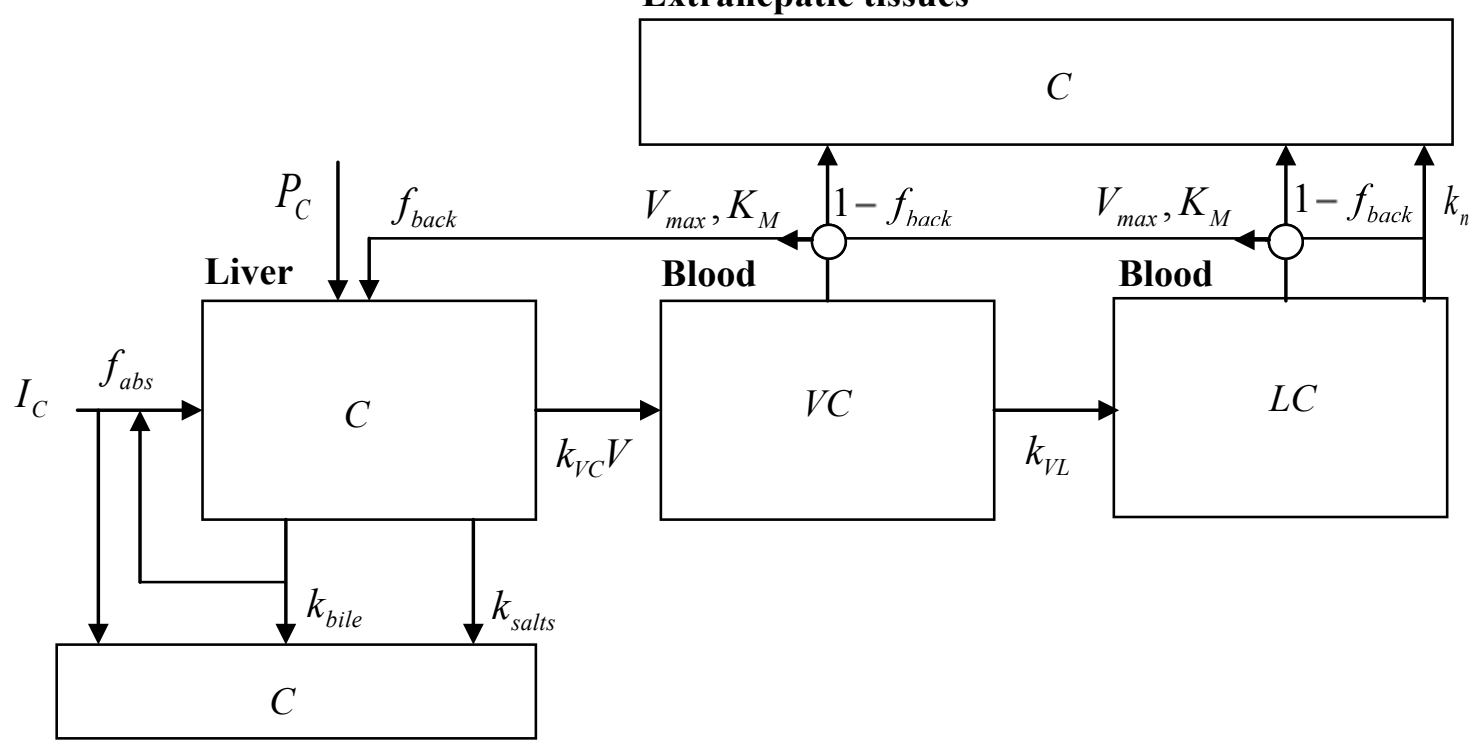

Faeces

Figure 1 Simplified scheme of LDL cholesterol metabolism in humans. For detailed description of the model see text. The definition of the model variables are summarised in Table 1. 
Table 1 Model variables and abbreviations used in the study

\begin{tabular}{|c|c|}
\hline Model variable & Abbreviation \\
\hline Endogenously produced cholesterol & $P_{C}$ \\
\hline Dietary cholesterol intake & $I_{C}$ \\
\hline External daily statin dose & $S$ \\
\hline External daily free phytosterol/-stanol dose & PS \\
\hline (Steady state) concentration of free cholesterol in the liver & C \\
\hline VLDL particles & V \\
\hline Absorbed cholesterol fraction & $f_{a b s}$ \\
\hline Fraction of produced VLDL cholesterol that re-enters the liver & $f_{\text {back }}$ \\
\hline Association rate of VLDL particles and free cholesterol to VLDL cholesterol & $k_{V C}$ \\
\hline Excretion of cholesterol from the cholesterol pool by bile & $k_{\text {bile }}$ \\
\hline Excretion of cholesterol through the formation of bile salts & $k_{\text {salts }}$ \\
\hline Reduction in cholesterol pool size & $R_{C}$ \\
\hline Cholesterol pool concentration in absence of statins and phytosterols/-stanols & $C_{0}$ \\
\hline Endogenous produced cholesterol in absence of statins and phytosterols/-stanols & $P_{C, 0}$ \\
\hline Uptake of dietary cholesterol in absence of statins and phytosterols/-stanols & $U_{C, 0}$ \\
\hline Absorbed cholesterol fraction in absence of statins and phytosterols/-stanols & $f_{a b s, 0}$ \\
\hline Reduction in endogenous cholesterol production & $R_{p}$ \\
\hline Reduction in fraction of cholesterol uptake from the diet & $R_{U}$ \\
\hline Ratio of exponential rates of different cholesterol elimination routes & $\rho_{k}$ \\
\hline VLDL cholesterol production rate & $P_{V C}$ \\
\hline Transformation rate of VLDL cholesterol to LDL cholesterol & $k_{V L}$ \\
\hline (Steady state) VLDL cholesterol concentration & VC \\
\hline Maximum rate of change in $(V) L D L$ cholesterol due to saturated uptake process & $V_{\max }$ \\
\hline Michaelis-Menten constant in (V)LDL cholesterol model & $K_{M}$ \\
\hline (Steady state) LDL cholesterol concentration & $L C$ \\
\hline LDL cholesterol production from VLDL cholesterol & $P_{L C}$ \\
\hline Clearance rate of $L D L$ cholesterol through non-saturated process & $k_{n}$ \\
\hline Maximal achievable reduction in endogenous cholesterol production & $R_{P, \max }$ \\
\hline Half maximum reduction statin dose & $S_{P, 1 / 2}$ \\
\hline Maximal achievable reduction in fraction of cholesterol uptake from the diet & $R_{U, \max }$ \\
\hline Half maximum reduction phytosterol/-stanol dose & $P S_{U, 1 / 2}$ \\
\hline
\end{tabular}

Moreover, up- and down-regulation of LDL receptors is not considered.

The model considers only steady state levels of cholesterol, VLDL cholesterol and LDL cholesterol. For a steady cholesterol level, the input of cholesterol should be balanced by its output. Clearance of cholesterol by VLDL cholesterol formation, bile excretion and bile salts formation is assumed to be non-saturated and is described by the product of clearance rates and steady cholesterol level.

Of the daily amount of VLDL cholesterol formation, $k_{V C} V \cdot C$, the product of steady cholesterol level $C$ with VLDL particles $V$ and association rate $k_{V C}$, a fraction $f_{\text {back }}$ is reabsorbed into the liver. It consists of VLDL cholesterol that is not used for LDL cholesterol production and of LDL cholesterol. The other fraction $1-f_{\text {back }}$ is taken up by the extrahepatic tissues, of which part is excreted through HDL cholesterol, which will not be considered in this modelling approach. As a consequence of this recycling, the effective clearance rate of cholesterol to VLDL cholesterol is $\left(1-f_{\text {back }}\right) \cdot k_{V C} V$. The amount of cholesterol eliminated through bile salts formation is $k_{\text {salt }} \cdot C$.

Likewise, because it is assumed that not only dietary cholesterol but also cholesterol cleared by bile with a daily amount of $k_{\text {bile }} \cdot C$ is reabsorbed through enterohepatic recycling, the effective clearance rate of cholesterol through bile is $\left(1-f_{a b s}\right) \cdot k_{\text {bile }}$.

As we consider the effect of statins and phytosterols/stanols on LDL cholesterol levels, the model becomes slightly more complicated. First, it is assumed that reduced cholesterol production $P_{C}$ is related to the external daily dose $S$ of statins, $P_{C}=P_{C}(S)$. Second, it is assumed that the reduced cholesterol fraction absorbed from dietary cholesterol intake relates to the amount of intake of phytosterols/-stanols (PS), $f_{a b s}=f_{a b s}(P S)$.

In the steady state the input cholesterol $P_{C}(S)+f_{a b s}$ $(P S) \cdot I_{C}$ is balanced by cleared cholesterol, which is the 
product of the effective clearance rates and the steady cholesterol level $\left(\left(1-f_{\text {back }}\right) k_{V C} V\right)+\left(1-f_{a b s}(P S)\right) k_{\text {bile }}$ $\left.+k_{\text {salts }}\right) \cdot C(S, P S)$. Thus, the steady cholesterol level is:

$$
C(S, P S)=\frac{P_{C}(S)+f_{a b s}(P S) \cdot I_{C}}{\left(1-f_{\text {back }}\right) \cdot k_{V C} V+\left(1-f_{\text {abs }}(P S)\right) \cdot k_{\text {bile }}+k_{\text {salts }}}(1)
$$

It should be noted that it is implicitly assumed that there is no interaction between statins and phytosterols/-stanols consumed, i.e. both compounds work simultaneously, independent of each other.

\section{Modelling of VLDL cholesterol level}

In the modelling of the cholesterol pool (equation (1)) it is assumed that the production of VLDL cholesterol $P_{V C}$ is proportional to both the concentration of VLDL particles and the free cholesterol level: $P_{V C}=k_{V C} V \cdot C(S, P S)$. Like for cholesterol, a steady state level $V C(S, P S)$ of VLDL cholesterol follows from the balance between its production and its clearance. VLDL cholesterol is assumed to be cleared due to the production of LDL cholesterol with daily clearance of $k_{V L} \cdot V C(S, P S)$ and due to saturated receptor-mediated uptake from blood into the liver and extrahepatic tissues [6]. Receptormediated uptake is assumed to follow Michaelis-Menten kinetics with a maximum clearance rate $V_{\max }$ and a saturation constant $K_{M}$.

Therefore, a steady state VLDL cholesterol level leads to the following mass balance for LDL cholesterol:

$$
P_{V C}=k_{V C} V \cdot C(S, P S)=k_{V L} \cdot V C(S, P S)+\frac{V_{\max } \cdot V C(S, P S)}{K_{M}+V C(S, P S)}
$$

The steady state VLDL cholesterol level can be obtained by solving the implicit equation for $V \mathrm{C}(S, P S)$. The explicit expression for $V C$ is deduced in Appendix 1. Note that of the Michaelis-Menten saturated clearance of VLDL cholesterol from blood a fraction $f_{\text {back }}$ goes into the liver. The complementary fraction 1- $f_{\text {back }}$ goes into extrahepatic tissues (Figure 1).

\section{Modelling of $L D L$ cholesterol level}

In the modelling of the VLDL cholesterol level (equation (2)), we assumed that the production of LDL cholesterol $P_{L C}$ is proportional to the steady VLDL cholesterol level: $P_{L C}=k_{V L} \cdot V C(S, P S)$. LDL cholesterol is assumed to be cleared with rate $k_{n}$ through a non-saturated process and by saturated uptake from blood into the liver and extrahepatic tissues by the same receptors as for the saturated uptake of VLDL cholesterol [6]. Hence, the mass balance for steady state LDL cholesterol is:

$$
P_{L C}=k_{V L} V C(S, P S)=k_{n} \cdot L C(S, P S)+\frac{V_{\max } \cdot L C(S, P S)}{K_{M}+L C(S, P S)}
$$

from which the LDL cholesterol level can be obtained by solving the implicit equation (3) for $L C(S, P S)$. The explicit expression for $L C$ can be found in Appendix 1.
Similar as for VLDL cholesterol, a fraction $f_{\text {back }}$ of the Michaelis-Menten saturated clearance of LDL cholesterol from blood goes into the liver. The complementary fraction 1- $f_{\text {back }}$ goes into extrahepatic tissues (Figure 1). $V_{\text {max }}, K_{M}$ are the same maximum elimination rate and saturation constant of the Michaelis-Menten saturated uptake of VLDL cholesterol from blood into the liver. These constants are assumed to be the same, but this assumption is not essential.

\section{Cholesterol reduction model \\ Modelling cholesterol reduction by statins and phytosterols/-stanols}

When it is assumed that statins and phytosterols/-stanols work independently of each other, the reduction in the cholesterol pool size can be expressed in terms of a reduction: $R_{P}(S)=P_{C}(S) / P_{C, 0}$ in cholesterol production caused by statin use and a reduction: $R_{U}(P S)$ $=f_{a b s}(P S) / f_{a b s, 0}$ in dietary cholesterol absorption across the gut wall caused by phytosterol/-stanol use. $P_{C, 0}$ and $f_{a b s, 0}$ denote the cholesterol production rate and absorption fraction in absence of statins or phytosterols/-stanols. From equation (1) it is derived in Appendix 2 that the corresponding reduction $R_{C}(S, P S)$ in the steady state cholesterol level is the product of the reduction due to a decrease in enterohepatic efficiency (first factor at the right side of equation (4)) and a weighted mean of the reduction due to a decrease in cholesterol production and uptake (second factor at the right side of equation (4)):

$$
R_{c}(S, P S)=\frac{C(S, P S)}{C_{0}}=\underbrace{\frac{\rho_{k}+1-f_{a b s, 0}}{\rho_{k}+1-f_{a b s}(P S)}}_{\text {first factor }} \times \underbrace{\left(\frac{\widetilde{R}_{p}(S)}{1+U_{C, 0} / P_{C, 0}}+\frac{\overbrace{\frac{R_{U}}{1+P_{C, 0}}\left(U_{C, 0}\right.}^{\text {first term }}}{1+P_{C}}\right)}_{\text {second factor }}
$$

Here, like for cholesterol production and absorption, $C_{0}$ and $U_{C, \text { o }}$ denote, respectively, the cholesterol pool concentration and dietary uptake in absence of statins and phytosterols/-stanols. In the first factor at the right side, the ratio $\rho_{k}$ denotes the proportion of cholesterol elimination through VLDL cholesterol production and bile salts production to cholesterol elimination through bile excretion, as introduced in Appendix 2.

The following remarks should be made regarding this model. First, the effectiveness of statins or phytosterols/stanols to lower cholesterol production is determined by the ratio of the contribution of endogenous produced cholesterol and the contribution of dietary cholesterol uptake to the cholesterol pool. Thus, when dietary cholesterol uptake is increased, the effectiveness of statins (first term in the second factor) is reduced with respect to the effectiveness of phytosterols/-stanols. Obviously, the opposite holds true for the effectiveness of phytosterols/-stanols. 
Second, the reduction in the absorbed fraction of cholesterol has an additional effect in total cholesterol pool reduction (the second term in the second factor at the right side of equation (4)) and a multiplicative one (first factor at the right side of equation (4)). The additional effect relates to the decrease of dietary cholesterol uptake reduction, whereas the multiplicative effect relates to the decrease in enterohepatic recycling efficiency, causing increased cholesterol elimination through bile.

Third, the additional reduction caused by statin and phystosterol/-stanol use is a weighted sum of the reduction in cholesterol production and cholesterol absorption because $1 /\left(1+P_{C, 0} / U_{C, 0}\right)+1 /\left(1+U_{C, 0} / P_{C, 0}\right)=1$.

Formulating reduction as a Michaelis-Menten process

In the model described above, we aim to associate reductions in the cholesterol pool size to reductions in LDL cholesterol level. In order to estimate the reduction in the cholesterol pool size following statin intake, $R_{P}(S)$, a reduction model has to be assumed. From experimental in vitro data from Shum et al [7]. a reasonable model assumption is obtained as follows. Shum et al. related the concentration of atorvastatin in plasma to the inhibition of the enzyme HMG-CoA reductase in vitro. We assumed a Michaelis-Menten saturated inhibition process. This model was fitted to their experimental data and provided a nearly perfect fit (Figure 2).

From this experimental in vitro result, it is proposed that the reduction in endogenously produced cholesterol is most likely Michaelis-Menten saturated with administered statin dose as well:

$$
R_{P}(S)=1-\frac{R_{P, \max } \cdot S}{S_{P, 1 / 2}+S}
$$

where $R_{P, \max } \leq 1$ determines the maximum achievable reduction and $S_{P, 1 / 2}$ is the half maximum reduction statin dose.

Based on the fact that cholesterol uptake is receptormediated,[8] we assumed that the reduction in the fraction absorbed cholesterol is like:

$$
R_{U}(P S)=1-\frac{R_{U, \max } \cdot P S}{P S_{U, 1 / 2}+P S}
$$

where $R_{U, \text { max }} \leq 1$ determines the maximum achievable reduction and $P S_{U, 1 / 2}$ is the half maximum reduction phytosterol/-stanol dose.

Thus, the total reduction in cholesterol pool size after combined intake of statins and phytosterols/-stanols is obtained by substituting equations (5) and (6) in equation (4):

$$
\begin{aligned}
R_{C}(S, P S)= & \frac{\rho_{k}+1-f_{a b s, 0}}{\rho_{k}+1-\left(1-R_{U, \max } \cdot P S /\left(P S_{U, 1 / 2}+P S\right)\right) \cdot f_{a b s, 0}} \\
& \times\left(1-\frac{P_{C, 0}}{P_{C, 0}+U_{C, 0}} \cdot \frac{R_{P, \max } \cdot S}{S_{P, 1 / 2}+S}-\frac{U_{C, 0}}{P_{C, 0}+U_{C, 0}} \cdot \frac{R_{U, \max } \cdot P S}{P S_{U, 1 / 2}+P S}\right)
\end{aligned}
$$

In this derivation we used $1 /\left(1+U_{C, 0} / P_{C, 0}\right)+1 /(1+$ $\left.P_{C, 0} / U_{C, 0}\right)=1$

\section{Parameter value estimation}

The reduced steady state cholesterol concentration can be obtained from a given daily dose of statins or phytosterols/-stanols by applying the reduction model

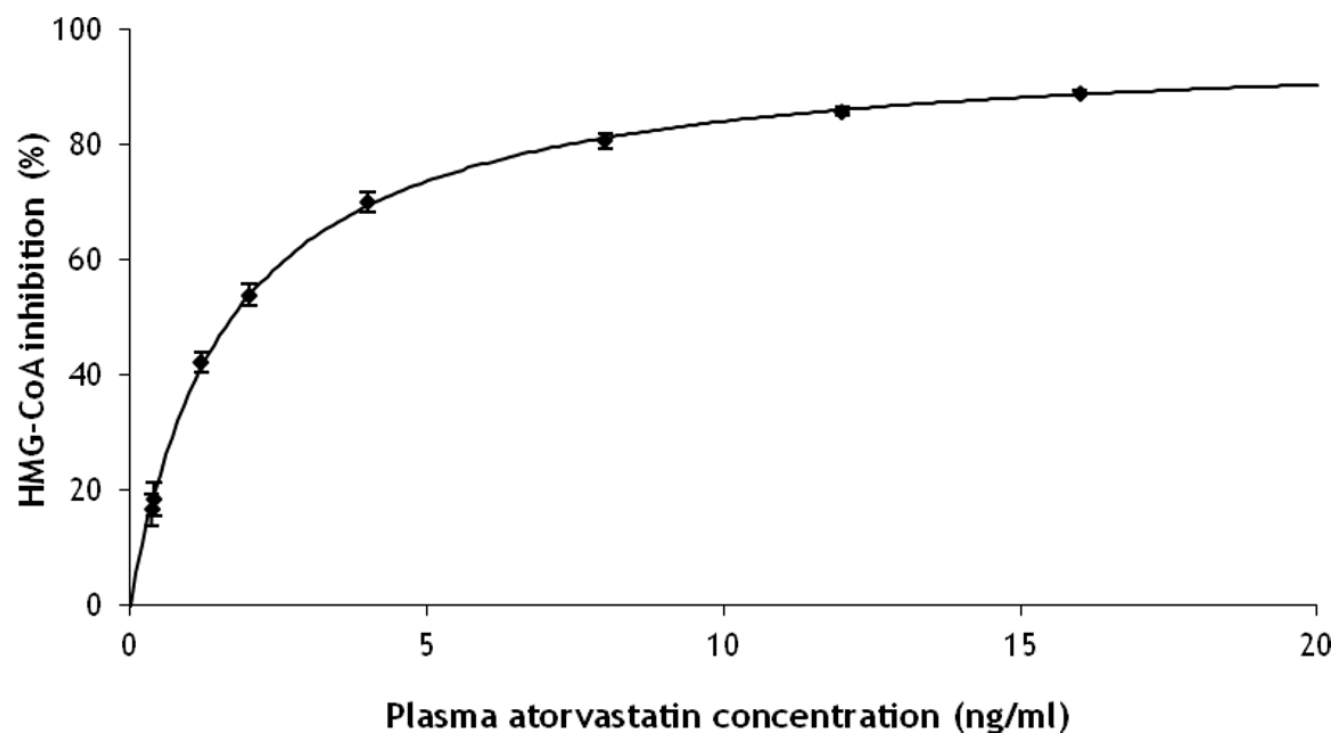

Figure 2 Simulated dose-response relation between in vitro plasma atorvastatin concentration ( $\mathrm{ng} / \mathrm{ml}$ ) and hydroxymethylglutarylcoenzyme A (HMG-CoA) inhibition (\%) in humans. Maximum inhibition is $97.8 \%$ and the concentration at half maximum inhibition is 1.64 $(\mathrm{ng} / \mathrm{ml})$. Solid symbols present data by Shum et al. [7] 
proposed in equation (7). From that reduced concentration, the reduced steady VLDL cholesterol level can be derived by solving equation (2), and subsequently the reduced LDL cholesterol level can be derived by solving equation (3). However, to be applicable in practice, model parameters should be known. In this section all parameters of our model are quantified based on data in the literature.

\section{Basic cholesterol model parameters}

It is assumed that the liver produces $P_{C, 0}=1000 \mathrm{mg}$ cholesterol per day [9-12]. Furthermore, we assumed a dietary cholesterol intake of $I_{C}=400 \mathrm{mg} / \mathrm{d}$, of which a fraction of $50 \%\left(f_{a b s, 0}=0.5\right)[9,13]$ is taken up in the liver $\left(U_{C, 0}=200 \mathrm{mg} / \mathrm{d}\right)$. The same fraction is supposed to be recycled through enterohepatic recycling of cholesterol excreted with bile. The final contribution to liver cholesterol input is assumed to be $70 \%$ of produced VLDL cholesterol $\left(f_{\text {back }}=0.7\right)$ [14].

It is assumed that the amount of cholesterol excreted with bile is $1000 \mathrm{mg} / \mathrm{d}[9,13,15]$ and consequently, 500 $\mathrm{mg} / \mathrm{d}$ re-enters the liver. The rate of excretion through the formation of bile salts is $400 \mathrm{mg} / \mathrm{d}$ [16]. Concerning the local liver balance, the input is 1000 (produced cholesterol, $P_{C, 0}$ ) plus 200 (uptake, $U_{C, 0}$ ) plus 500 (recycled, $\left(1-f_{a b s, 0}\right) \cdot k_{b i l e} \cdot C_{0}$ ) plus 700 (back transport, $\left.f_{\text {back }} \cdot k_{V C} V \cdot C_{0}\right)$ making a total of $2400 \mathrm{mg} / \mathrm{d}$. The output is 1000 (bile, $k_{\text {bile }} \cdot C_{0}$ ) plus 400 (bile salts, $k_{\text {salts }} \cdot C_{0}$ ), and making a total of $2400 \mathrm{mg} / \mathrm{d}$, plus the production of $1000 \mathrm{mg}$ VLDL cholesterol per day (VLDL cholesterol, $\left.k_{V C} V \cdot C_{0}\right)$. Moreover, as the elimination from the liver is proportional to the production rates of bile salts, cholesterol in bile and VLDL cholesterol, the ratio of these production rates is $k_{\text {salts }}: k_{\text {bile }}: k_{V C} V=0.4: 1: 1$.

From Sahlin et al. $[17,18]$ we estimated the free cholesterol content in liver to be $55 \mathrm{nmol} / \mathrm{mg}$ microsomal protein. Together with a microsomal protein content of $45 \mathrm{mg} / \mathrm{g}$ liver[19] this amounts to $2500 \mu \mathrm{mol} / \mathrm{kg}$ liver which equals $960 \mathrm{mg}$ free cholesterol/ $\mathrm{kg}$ liver. From this free cholesterol concentration and the daily bile excretion, one can derive $k_{\text {bile }}=1000 / 960=1.04, k_{V C} V=$ 1.04 and $k_{\text {salts }}=0.416$.

Dietschy et al. [6] report LDL cholesterol model parameter values in humans. When assuming a subject of 70 $\mathrm{kg}$, these values are $V_{\max }=1340 \mathrm{mg} / \mathrm{d}, K_{M}=90 \mathrm{mg} / \mathrm{dl}$, $k_{n}=5 \mathrm{dl} / \mathrm{d}$ and $P_{L C}=910 \mathrm{mg} / \mathrm{d}$. Based on these values, a steady cholesterol level $L C=67 \mathrm{mg} / \mathrm{dl}$ results from equation (3).

The VLDL:LDL:HDL cholesterol ratio was estimated to be 1:8:3 [20]. Thus the corresponding VLDL level is $8.4 \mathrm{mg} / \mathrm{dl}$. As the LDL cholesterol production rate is equal to $k_{V L} \cdot V C$ (equation (3)), $k_{V L}=108(\mathrm{dl} / \mathrm{d})$. From equation (2) a VLDL cholesterol production of 1020 $\mathrm{mg} / \mathrm{d}$ can be calculated. Above it is assumed to be 1000 $\mathrm{mg} / \mathrm{d}$ which shows a consistency error of $2 \%$ only.

\section{Cholesterol reduction model parameters}

From the ratio between the effective clearances, introduced in the basic cholesterol model parameters section, one can derive that the ratio $\rho_{k}$ in equation (4) is 0.7 . The four remaining parameters $R_{P, \max }, S_{P, 1 / 2}, R_{U \text {, max }}$, $\mathrm{PS}_{U, 1 / 2}$ are unknown and were fitted to optimise their likelihood in comparing modelled LDL cholesterol reduction induced by cholesterol reduction to LDL cholesterol reduction data. Thus, given an estimation of the four cholesterol reduction model parameters, the reduction in steady state cholesterol is calculated, the resulting reduction in VLDL cholesterol is determined from equation (2) and the resulting reduction in LDL cholesterol is determined from equation (3).

To simulate the appropriateness of our model, reduced levels are compared with the experimental data for separate intakes of atorvastatin[21] and phytosterols/-stanols [22]. For this procedure we use for $R_{P, \text { max }}$, $S_{P, 1 / 2}$ data from a recent meta-analysis of Berry et al. [21] that shows experimentally determined in vivo LDL cholesterol reduction due to atorvastatin dose. For $R_{U}$, max $P S_{U, 1 / 2}$ we use data presented in Demonty et al. [22] showing experimentally determined in vivo LDL cholesterol reduction due to intake of free phytosterols/stanols, i.e. phytosterols/-stanols not in esterified form. Finally, we simulate reductions after combined intake of atorvastatin and phytosterols/-stanols.

\section{Results}

Separate intake

LDL cholesterol reduction by atorvastatin

We applied equation (7) together with the corresponding VLDL and LDL cholesterol levels equations (2) and (3) to data in Berry et al. [21] in a LDL cholesterol reduction model using the model parameters given above. The unknown parameter values in equation (7) were estimated through fitting the maximum reduction $R_{P, \max }$ and the statin dose $S_{P, 1 / 2}$ when half maximum reduction is reached.

Figure 3 shows the comparison of the resulting LDL cholesterol reduction model to the data in Berry et al. [21] Parameter values are $R_{P, \max }=0.544$ (standard error $(\mathrm{SE})=0.033)$ and half reduction dose $S_{P, 1 / 2}=6.7(\mathrm{SE}=$ 1.4) $\mathrm{mg} / \mathrm{d}$. Given the parsimony of dose levels and scattering of data, a good comparison between the model and the experimental results is obtained $\left(R^{2}=0.70\right)$.

\section{LDL cholesterol reduction by phytosterols/-stanols}

We applied equation (7) together with the corresponding VLDL and LDL cholesterol levels equations (2) and (3) to data in Demonty et al. [22] in a LDL cholesterol reduction model. The unknown parameter values in equation (7) were estimated through fitting the maximum reduction $R_{U, \max }$ and the free phytosterol/-stanol dose $P S_{U, 1 / 2}$ when half maximum reduction is reached. 


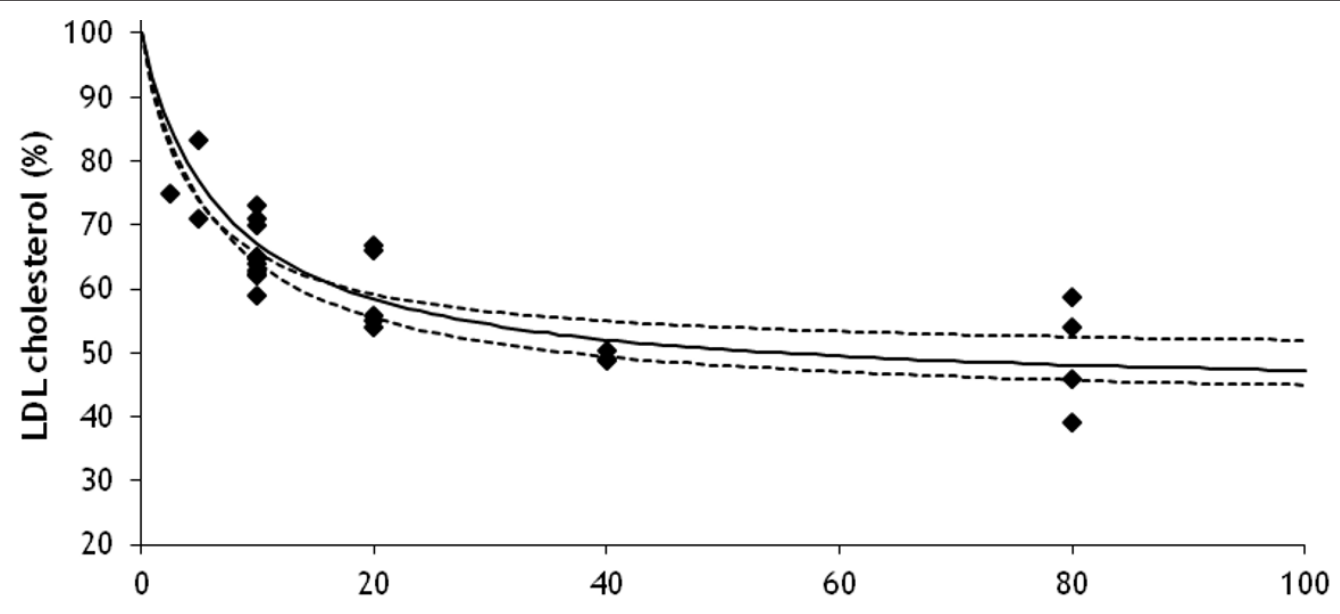

Atorvastatin dose $(\mathrm{mg} / \mathrm{d})$

Figure 3 Simulated reduction (\%) in LDL cholesterol after treatment with different doses of atorvastatin. The solid line shows the fit to the model and symbols represent experimental data from Berry et al. [21] Values for the Michaelis-Menten parameters are: effective maximum $\mathrm{LDL}$ cholesterol reduction $\left(R_{P, \max }\right)=0.544$ and half maximum reduction statin dose $\left(S_{p, 1 / 2}\right)=6.7 \mathrm{mg} / \mathrm{d}$. The dashed lines show the $5 \%$ and $95 \%$ uncertainty range in reduction obtained by correlated sampling (correlation coefficient $\rho=0.88$ ) of $R_{P, \max }$ and $S_{P, 1 / 2}$ from their covariance matrix.

Figure 4 shows the comparison of the resulting LDL cholesterol reduction model to the data of Demonty et al. [22] Parameter values are $R_{U, \max }=0.221(\mathrm{SE}=$ $0.039)$ and half reduction dose $P S_{U, 1 / 2}=1.78$ ( $\mathrm{SE}=$ $0.69) \mathrm{mg} / \mathrm{d}$. The model shows reasonable agreement with the published experimental data $\left(\mathrm{R}^{2}=0.17\right)$.

\section{Combined intake}

LDL cholesterol reduction by combined use of atorvastatin and phytosterols/-stanols

The model is applied to LDL cholesterol reduction due to the combined intake of statins and phytosterols/-stanols. For subjects with a daily intake of $0,20,40$ and 80

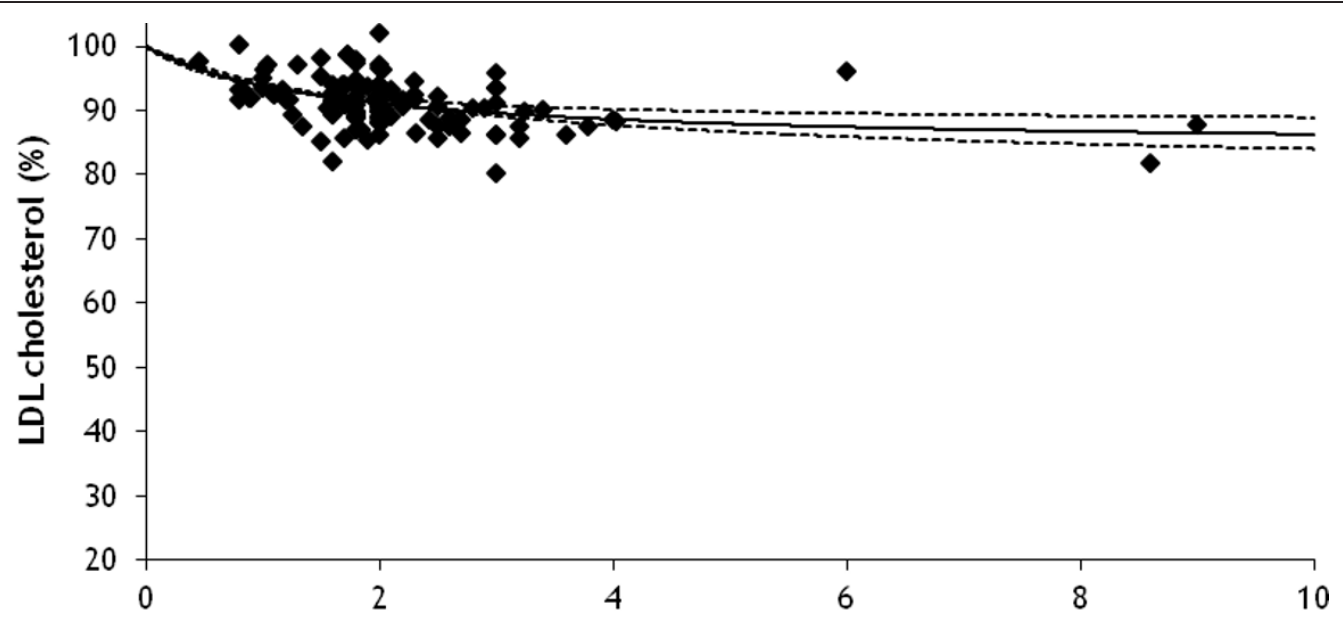

Phytosterol/-stanol dose (g/d)

Figure 4 Simulated reduction (\%) in LDL cholesterol after treatment with different doses of phytosterols/-stanols. The solid line shows the fit to the model and symbols represent experimental data from Demonty et al. [22] Values for the Michaelis-Menten parameters are: effective maximum $L D L$ cholesterol reduction $\left(R_{U, \max }\right)=0.221$ and half maximum reduction phytosterol/-stanol dose $\left(P S_{U}, 1 / 2\right)=1.78 \mathrm{mg} / \mathrm{d}$. The dashed lines show the $5 \%$ and $95 \%$ uncertainty range in reduction obtained by correlated sampling (correlation coefficient $\rho=0.98$ ) of $R_{U}$, max and $P S_{U, 1 / 2}$ from their covariance matrix. 
mg atorvastatin, respectively, we show in Figure 5 the total reduction in LDL cholesterol as a function of daily phytosterol/-stanol intake. For the daily recommended intake level of $2 \mathrm{~g}$ free phytosterols/-stanols (equivalent to $3.3 \mathrm{~g} / \mathrm{d}$ phytosterol/-stanol esters), the additional reduction is $4.2 \%$ for a daily statin dose of $80 \mathrm{mg}, 4.5 \%$ for a daily statin dose of $40 \mathrm{mg}, 4.8 \%$ for a daily statin dose of $20 \mathrm{mg}$ and $7.8 \%$ with no statin intake. Thus, the reduction in LDL cholesterol caused by additional phytosterol/-stanol intake decreases with increasing daily atorvastatin dose. However, when considering the additional decrease as a percentage of the LDL cholesterol level already reduced due to statin intake, the additional decrease ranges from $7.8 \%$ (no statin intake) to $8.6 \%$ (80 mg daily statin dose). Randomised controlled trials in which patients on statin therapy were treated daily with 1.8 to $6 \mathrm{~g}$ phytosterol or -stanol esters have shown reductions in the same order of magnitude, i.e. between $6.1 \%$ and $10.3 \%$ [23-28].

The reduction in enterohepatic recycling contributes for $68 \%, 58 \%, 56 \%$ and $55 \%$ of the total decrease in LDL cholesterol levels at daily statin doses of 0, 20, 40 and $80 \mathrm{mg}$, respectively. At the same recommended phytosterol/-stanol intake level of $2 \mathrm{~g} / \mathrm{d}$, the additional decrease in LDL cholesterol by physterols/-stanols for a daily statin dose of $20 \mathrm{mg}$ (4.8\%) is equal to the additional decrease by doubling daily statin dose to $40 \mathrm{mg}$ (5.3\%). For a daily statin dose of $40 \mathrm{mg}$ the additional decrease in LDL cholesterol by physterols/-stanols $(4.5 \%)$ is $30 \%$ larger than the additional decrease by doubling daily statin dose to $80 \mathrm{mg}(3.2 \%)$.

\section{Discussion}

In this paper, a mathematical model is presented that simulates the reductions in LDL cholesterol after separate and combined intake of atorvastatin and phytosterols/-stanols in humans. We demonstrated that a daily intake of $2 \mathrm{~g}$ phytosterols/-stanols reduces LDL cholesterol level by about $8 \%$ to $9 \%$ on top of the reduction resulting from statin use. This level of reduction is consistent with the findings of randomised controlled trials [23-28]. The additional decrease in LDL cholesterol caused by phytosterol/-stanol use at the recommended level of $2 \mathrm{~g} / \mathrm{d}$ appeared to be similar or even greater than the decrease achieved by doubling the statin dose, a finding that has been observed previously in human trials [23,29]. The reduction in LDL cholesterol level due to phytosterol/-stanol use results from a decrease in the intestinal uptake of dietary cholesterol (additional effect) and a reduction in enterohepatic recycling (multiplicative effect). For daily statin doses of $20 \mathrm{mg}$ or more, the contribution of the enterohepatic recycling reduction is $55 \%$ or more. When no statin is used, this contribution is $68 \%$.

Mathematical models provide a valuable means of interpreting experimental data and improving the ability to predict the response to a given treatment. Other modelling studies have focused on cholesterol metabolism, but are merely aimed at answering questions on the cellular level or tend to focus on specific areas of cholesterol metabolism, such as the fluid dynamics of lipid accumulation on the arterial wall or the chemical kinetics of LDL oxidation [30-32].

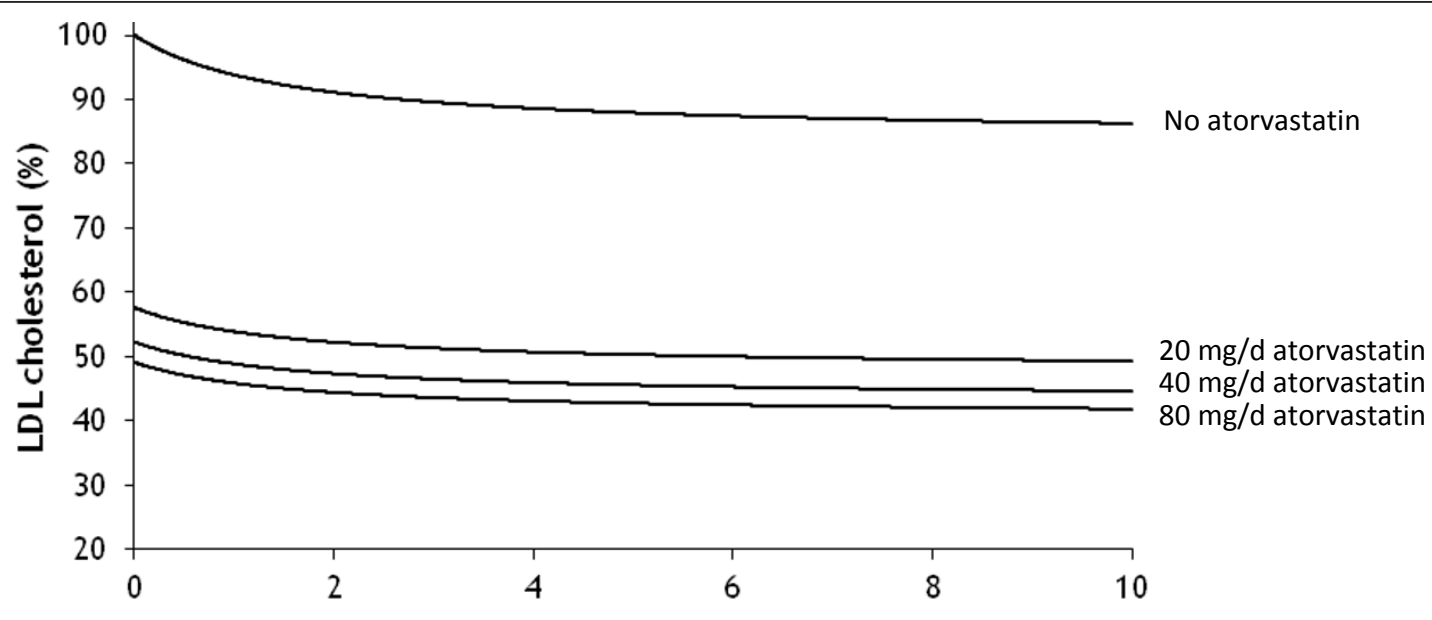

Phytosterol/-stanol dose (g/d)

Figure 5 Simulated reduction (\%) in LDL cholesterol after combined treatment with different doses of free phytosterols/-stanols and atorvastatin in humans. The lines from upper to lower show LDL cholesterol reduction for subjects that are exposed to no atorvastatin, or daily doses of 20,40 or $80 \mathrm{mg}$ atorvastatin, respectively. 
In the present study, the separate and combined effects of the cholesterol-lowering drug atorvastatin and functional foods with phytosterols/-stanols in humans were modelled. Yet, this model can easily be applied to other statins and similar acting (functional) foods as well. Products with soluble dietary fibres, for example, are also known to lower total and LDL cholesterol by reducing the intestinal (re)absorption of cholesterol and bile acids, although they work by a different mechanism as phytosterols/-stanols [4,33,34]. Moreover, individuals' specific reductions in total and LDL cholesterol can be predicted, based on certain genetic variants. For example, the ratio of cholesterol synthesis to cholesterol absorption varies between individuals and is an important determinant for the cholesterol pool size [35]. Also mutations in the LDL receptor gene causing familial hypercholesterolaemia can be modelled by varying the parameter $V_{\text {max }}$.

There are a few possible directions for improving our model. First, the model could be extended by including the up- and down regulatory mechanisms involving the LDL receptors. Nonetheless, since we assumed that the clearing of (V)LDL cholesterol from the blood follows Michaelis-Menten kinetics, we implicitly included receptor-mediated uptake in the model. Also other regulatory control pathways were disregarded, such as the existence of a hepatic cholesteryl ester pool that might be involved in cholesterol homeostasis and the regulatory loop in the synthesis of LDL receptors [30]. Another extension would include reverse cholesterol transport mediated by HDL [20]. Moreover, the proposed model assumes that the reducing effects of statins and functional foods are independent of each other. Although this is likely the case for the combination of phytosterols/-stanols and statins, [22,24,36,37] it is uncertain whether this applies for other food-drug combinations. It has, for example, been proposed that soluble dietary fibres reduce the intestinal uptake of statins [38,39]. Our model should be extended to include such an interaction.

In conclusion, we proposed a simplified mathematical model to simulate the reduction in LDL cholesterol after separate and combined intake of statins and functional foods acting on intestinal (re)absorption of cholesterol or bile acids in humans. In future work, this model can be extended to include more complex (regulatory) mechanisms.

\section{Appendix 1}

\section{Steady VLDL cholesterol concentration}

The mass balance equation (2) can be rewritten as a quadratic equation in $V C$ :

$$
k_{V L} \cdot V C^{2}+\left(V_{\max }+k_{V L} \cdot K_{M}-k_{V C} V \cdot C\right) \cdot V C-K_{M} \cdot k_{V C} V \cdot C=0
$$

Here, we suppress the dependencies on statin administration $S$ and dietary phytosterol/-stanol intake PS.

A quadratic equation has two solutions, but the only physicochemical relevant solution for which the VLDL cholesterol concentration is non-negative is:

$$
\begin{aligned}
V C & =\frac{1}{2 k_{V L}}\left(k_{V C} V \cdot C-k_{V L} \cdot K_{M}-V_{\max }\right. \\
& \left.+\sqrt{\left(k_{V C} V \cdot C-k_{V L} \cdot K_{M}-V_{\max }\right)^{2}+4 k_{V L} \cdot K_{M} \cdot k_{V C} V \cdot C}\right)
\end{aligned}
$$

\section{Steady LDL cholesterol concentration}

Like for VLDL cholesterol, the mass balance equation

(3) can be rewritten as:

$$
k_{n} \cdot L C^{2}+\left(V_{\max }+k_{n} \cdot K_{M}-k_{V L} \cdot V C\right) \cdot L C-K_{M} \cdot k_{V L} \cdot V C=0
$$

with as solution:

$$
\begin{aligned}
L C & =\frac{1}{2 k_{n}}\left(k_{V L} \cdot V C-k_{n} \cdot K_{M}-V_{\max }\right. \\
& \left.+\sqrt{\left(k_{V L} \cdot V C-k_{n} \cdot K_{M}-V_{\max }\right)^{2}+4 k_{n} \cdot K_{M} \cdot k_{V L} \cdot V C}\right)
\end{aligned}
$$

\section{Appendix 2}

Reduction in steady state cholesterol

The reduction in steady state cholesterol level is:

$$
\begin{aligned}
R_{C}(S, P S) & =\frac{C(S, P S)}{C_{0}} \\
& =\frac{\left(P_{C}(S)+f_{a b s}(P S) \cdot I_{C}\right) /\left(\left(1-f_{b a c k}\right) \cdot k_{V C} V+\left(1-f_{a b s}(P S)\right) \cdot k_{b i l e}+k_{\text {salts }}\right)}{\left(P_{C, 0}+f_{a b s, 0} \cdot I_{C}\right) /\left(\left(1-f_{\text {back }}\right) \cdot k_{V C} V+\left(1-f_{a b s, 0}\right) \cdot k_{b i l e}+k_{\text {salts }}\right)} \\
& =\frac{\left(1-f_{\text {back }}\right) \cdot k_{V C} V+\left(1-f_{a b s, 0}\right) \cdot k_{b i l e}+k_{\text {salts }}}{\left(1-f_{\text {back }}\right) \cdot k_{V C} V+\left(1-f_{a b s}(P S)\right) \cdot k_{\text {bile }}+k_{\text {salts }}} \cdot \frac{P_{C}(S)+f_{a b s}(P S) \cdot I_{C}}{P_{C, 0}+f_{a b s, 0} \cdot I_{C}} \\
& =\frac{\rho_{k}+1-f_{a b s, 0}}{\rho_{k}+1-f_{a b s}(P S)} \cdot\left(\frac{P_{C}(S)}{P_{C, 0}+U_{C, 0}}+\frac{f_{a b s}(P S) \cdot I_{C}}{P_{C, 0}+f_{a b s, 0} \cdot I_{C}}\right) \\
& =\frac{\rho_{k}+1-f_{a b s, 0}}{\rho_{k}+1-f_{a b s}(P S)} \cdot\left(\frac{R_{P}(S)}{1+U_{C, 0} / P_{C, 0}}+\frac{R_{U}(P S)}{1+P_{C, 0} / U_{C, 0}}\right)
\end{aligned}
$$

In the third line, the ratio of clearance rates $\rho_{k}=((1-$ $\left.\left.f_{\text {back }}\right) \cdot k_{V C} V+k_{\text {salts }}\right) / k_{\text {bile }}$ is introduced. In this line also one instance of $f_{a b s, 0} \cdot I_{C}$ is substituted by $U_{C, 0}$. In the fourth line the definition of production reduction and, after dividing out intake $I_{C}$, of uptake reduction is substituted.

\section{Acknowledgements}

The authors thank Jeljer Hoekstra for detailed and helpful comments on earlier drafts of this manuscript.

\section{Author details}

${ }^{1}$ Division of Pharmacoepidemiology and Clinical Pharmacology, Utrecht Institute for Pharmaceutical Sciences, Utrecht University, P.O. Box 80082, 3508 TB Utrecht, The Netherlands. ${ }^{2}$ National Institute for Public Health and the Environment, P.O. Box 1, 3720 BA Bilthoven, The Netherlands. 


\section{Authors' contributions}

SE and JVE developed, tested and validated the model, and drafted the first version of the manuscript. Subsequent versions of the report were written by SE and JvE with input and critical revisions by all authors. All authors have read and approved the final manuscript as submitted.

\section{Competing interests}

The authors declare that they have no competing interests.

Received: 31 August 2011 Accepted: 21 October 2011

Published: 21 October 2011

\section{References}

1. Randomised trial of cholesterol lowering in $\mathbf{4 4 4 4}$ patients with coronary heart disease: the Scandinavian Simvastatin Survival Study (4S). Lancet 1994, 344(8934):1383-1389

2. Baigent C, Keech A, Kearney PM, Blackwell L, Buck G, Pollicino C, Kirby A, Sourjina T, Peto R, Collins R, Simes R: Efficacy and safety of cholesterollowering treatment: prospective meta-analysis of data from 90,056 participants in 14 randomised trials of statins. Lancet 2005, 366(9493):1267-78.

3. Amarenco P, Labreuche J, Lavalle P, Touboul P: Statins in stroke prevention and carotid atherosclerosis: systematic review and up-todate meta-analysis. Stroke 2004, 35(12):2902-2909.

4. Plat J, Mensink RP: Plant stanol and sterol esters in the control of blood cholesterol levels: mechanism and safety aspects. Am J Cardiol 2005, 96(1A):15D-22D.

5. Marinangeli CP, Varady KA, Jones PJ: Plant sterols combined with exercise for the treatment of hypercholesterolemia: overview of independent and synergistic mechanisms of action. J Nutr Biochem 2006, 17(4):217-24.

6. Dietschy JM, Turley SD, Spady DK: Role of liver in the maintenance of cholesterol and low density lipoprotein homeostasis in different animal species, including humans. J Lipid Res 1993, 34(10):1637-59.

7. Shum YY, Huang N, Walter G, Black A, Sekerke C, Chang T, Whitfield LR: Development, validation, and interlaboratory comparison of an HMGCoA reductase inhibition assay for quantitation of atorvastatin in plasma matrices. Ther Drug Monit 1998, 20(1):41-49.

8. Davis H, Altmann S: Niemann-Pick C1 Like 1 (NPC1L1) an intestinal sterol transporter. Biochim Biophys Acta 2009, 1791(7):679-683.

9. McNamara DJ: Effects of fat-modified diets on cholesterol and lipoprotein metabolism. Annu Rev Nutr 1987, 7:273-290.

10. Rajaratnam RA, Gylling H, Miettinen TA: Cholesterol absorption, synthesis, and fecal output in postmenopausal women with and without coronary artery disease. Arterioscler Thromb Vasc Biol 2001, 21(10):1650-1655.

11. Insel P, Ross D, McMahon K, Bernstein M, (eds): Nutrition. Jones and Bartlett Publishers, Sudbury, MA; 2011.

12. Stipanuk MH, (ed): Biochemical and physiological aspects of human nutrition. Lipid metabolism-Synthesis and oxidation. W.B. Saunders Co., Philadelphia, USA; 2000.

13. van der Velde A, Brufau G, Groen A: Transintestinal cholesterol efflux. Curr Opin Lipidol 2010, 21(3):167-171.

14. Demirezen EM, Barlas Y: A simulation model for blood cholesterol dynamics and related disorders. 2009, 2011(12 July):1-38.

15. Phillips GB: The lipid composition of human bile. Biochim Biophys Acta 1960, 41:361-363.

16. Bisschop P, Bandsma RHJ, Stellaard F, Harmsel A, Meijer A, Sauerwein H, Kuipers F, Romijn J: Low-fat, high-carbohydrate and high-fat, lowcarbohydrate diets decrease primary bile acid synthesis in humans. Am J Clin Nutr 2004, 79(4):570-576.

17. Sahlin S, Granstrm L, Gustafsson U, Sthlberg D, Backman L, Einarsson K: Hepatic esterification rate of cholesterol and biliary lipids in human obesity. J Lipid Res 1994, 35(3):484-490.

18. Sahlin S, Sthlberg D, Einarsson K: Cholesterol metabolism in liver and gallbladder mucosa of patients with cholesterolosis. Hepatology 1995, 21(5):1269-1275

19. Houston JB: Utility of in vitro drug metabolism data in predicting in vivo metabolic clearance. Biochem Pharmacol 1994, 47(9):1469-1479.

20. Kumar PJ, Clark ML: Clinical medicine - Diabetes Mellitus and other disorders of metabolism. W.B. Saunders 2002.
21. Berry DA, Berry SM, McKellar J, Pearson TA: Comparison of the doseresponse relationships of 2 lipid-lowering agents: a Bayesian metaanalysis. Am Heart J 2003, 145(6):1036-1045.

22. Demonty I, Ras RT, van der Knaap HC, Duchateau GS, Meijer L, Zock PL, Geleijnse JM, Trautwein EA: Continuous dose-response relationship of the LDL-cholesterol-lowering effect of phytosterol intake. J Nutr 2009, 139(2):271-84.

23. Blair SN, Capuzzi DM, Gottlieb SO, Nguyen T, Morgan JM, Cater NB: Incremental reduction of serum total cholesterol and low-density lipoprotein cholesterol with the addition of plant stanol ester-containing spread to statin therapy. Am J Cardiol 2000, 86(1):46-52.

24. Simons LA: Additive effect of plant sterol-ester margarine and cerivastatin in lowering low-density lipoprotein cholesterol in primary hypercholesterolemia. Am J Cardiol 2002, 90(7):737-40.

25. Richter WO: Treatment of severe hypercholesterolemia with a combination of beta-sitosterol and lovastatin. Curr Ther Res 1996, 57(7):497-505, 497

26. Castro Cabezas M, de Vries JH, Van Oostrom AJ, lestra J, van Staveren WA: Effects of a stanol-enriched diet on plasma cholesterol and triglycerides in patients treated with statins. J Am Diet Assoc 2006, 106(10):1564-9.

27. Goldberg AC, E OR Jr, Bateman JH, Schimmoeller L, McPherson TB, Spilburg CA: Effect of plant stanol tablets on low-density lipoprotein cholesterol lowering in patients on statin drugs. Am J Cardiol 2006, 97(3):376-9.

28. De Jong A, Plat J, Bast A, Godschalk RW, Basu S, Mensink RP: Effects of plant sterol and stanol ester consumption on lipid metabolism, antioxidant status and markers of oxidative stress, endothelial function and low-grade inflammation in patients on current statin treatment. Eur J Clin Nutr 2008, 62:263-73.

29. Katan MB, Grundy SM, Jones P, Law M, Miettinen T, Paoletti R: Efficacy and safety of plant stanols and sterols in the management of blood cholesterol levels. Mayo Clin Proc 2003, 78(8):965-78.

30. August $\mathrm{E}$, Parker $\mathrm{KH}$, Barahona $\mathrm{M}$ : A dynamical model of lipoprotein metabolism. Bull Math Biol 2007, 69(4):1233-1254.

31. Chun PW, Espinosa AJ, Lee CW, Shireman RB, Brumbaugh EE: Low density lipoprotein receptor regulation. Kinetic models. Biophys Chem 1985, 21(34):185-209.

32. Wattis JAD, O'Malley B, Blackburn H, Pickersgill L, Panovska J, Byrne HM, Jackson KG: Mathematical model for low density lipoprotein (LDL) endocytosis by hepatocytes. Bull Math Biol 2008, 70(8):2303-2333.

33. Theuwissen E, Mensink RP: Water-soluble dietary fibers and cardiovascular disease. Physiol Behav 2008, 94(2):285-92.

34. Eussen $\mathrm{S}$, Klungel $\mathrm{O}$, Garssen J, Verhagen $\mathrm{H}$, van Kranen $\mathrm{H}$, van Loveren $\mathrm{H}$, Rompelberg C: Support of drug therapy using functional foods and dietary supplements: focus on statin therapy. Br I Nutr 2010, 103(9):1260-1277.

35. Gylling $H$, Miettinen TA: Baseline intestinal absorption and synthesis of cholesterol regulate its response to hypolipidaemic treatments in coronary patients. Atherosclerosis 2002, 160(2):477-81.

36. Eussen S, de Jong N, Rompelberg C, Garssen J, Verschuren W, Klungel O: Dose-dependent cholesterol-lowering effects of phytosterol/phytostanolenriched margarine in statin users and statin non-users under free-living conditions. Public Health Nutr 2011, 1-10.

37. de Jong N, Zuur A, Wolfs MC, Wendel-Vos GC, van Raaij JM, Schuit AJ Exposure and effectiveness of phytosterol/-stanol-enriched margarines. Eur J Clin Nutr 2007, 61:1407-15.

38. Richter WO, Jacob BG, Schwandt P: Interaction between fibre and lovastatin. Lancet 1991, 338(8768):706.

39. Eussen SRBM, Rompelberg CJM, Andersson K, Klungel O, Hellstrand P, Oste R, van Kranen H, Garssen J: Simultaneous intake of oat bran and atorvastatin reduces their efficacy to lower lipid levels and atherosclerosis in LDLr-/- mice. Pharmacological research 2011, 64(1):36-43.

doi:10.1186/1476-511X-10-187

Cite this article as: Eussen et al:: Modelling approach to simulate reductions in LDL cholesterol levels after combined intake of statins and phytosterols/-stanols in humans. Lipids in Health and Disease 2011 10:187. 